\title{
SINGULARITIES, DEFECTS AND CHAOS IN ORGANIZED FLUIDS
}

\author{
ROLAND RIBOTTA, AHMED BELAIDI and ALAIN JOETS \\ Laboratoire de Physique des Solides \\ Université Paris-sud, 91405 Orsay, France \\ E-mail: ribotta@lps.u-psud.fr
}

\begin{abstract}
The singularities occurring in any sort of ordering are known in physics as defects. In an organized fluid defects may occur both at microscopic (molecular) and at macroscopic scales when hydrodynamic ordered structures are developed. Such a fluid system serves as a model for the study of the evolution towards a strong disorder (chaos) and it is found that the singularities play an important role in the nature of the chaos. Moreover both types of defects become coupled at the onset of turbulence. Besides this specificity, the results can be generalized to any structured physical system. They tend to demonstrate that the full knowledge of the system is "contained inside the surroundings of the singularity". It is also shown that such defects play a crucial role in all types of transitions between homogeneously ordered states from the rest state up to chaos.
\end{abstract}

1. Introduction. All the physical systems show a large variety of examples of singularities in some field which represents either the structure of the system itself or its main physical properties. For instance, a physical state may be characterized by its degree of ordering and thus, defects of any type of ordering are considered as singularities. The degree of ordering at any scale is usually a complex quantity (the "order-parameter") and is at first, taken as continuous over the relevant space. In that simple case the state is said uniform or homogeneous. In such a state, a singularity is associated with a discontinuity of the order-parameter at some well defined place. However, not all physical discontinuities are singular and in the following we shall be concerned mainly with topological singularities and only mention the existence of non-singular discontinuities. In fact, physics never admit such ideal discontinuities and there exists a small area surrounding the defect that insures a connection with the order-parameter value at large scales. This area is of prime importance because most often, singularities are topologically unstable. Such an area results from the constraint applied on the system to maintain, at least

2000 Mathematics Subject Classification: Primary 46C20; Secondary 32G81.

Key words and phrases: order, defects, chaos, topology, homotopy groups.

The paper is in final form and no version of it will be published elsewhere. 
transiently, the existence of the singularity. One uses to say that the physics describing the whole state is "contained" inside this area. Physical as well as topological descriptions of various defects have been made extensively for the last decades in various media ranging from solids ([15], [18], [20]) to biological materials [2]. In metallurgy it is known that most of the macroscopic properties of solids depend strongly on the density and the dynamics of the defects. However the precise description of individual defects was lacking certainly because of the impossibility to observe them directly at sufficiently small scales. Therefore their structure and dynamics have been for long times rather considered from a speculative or academic point of view. On the other hand, the fact that hydrodynamic structures occur on macroscopic scales, has made possible the direct and thus accurate study of both the topology and the role of the defects in such model systems.

In the 70's G. Toulouse, M. Kléman and L. Michel ([14]-[16], [26]) developed a systematic topological analysis to distortions in ordered media in various physical systems. In particular they showed that the defects can be classified according to their homotopy group. On the other hand the role of defect as regards their influence on some physical and mechanical properties of solids is well-documented (see for instance [20]), even if the mechanisms involved are not yet fully understood. However, their role has only recently been considered in the transitions between thermodynamic phases. Even though defects in structures arising in out-of-equilibrium systems were observed since the beginning, it is only in the 80 's that they received a careful attention. Nowadays and from these studies one understands that singularities play a key role not only in the phase changes (symmetry-breakings) but also at the onset of chaos. Further we show that, acting on the defects in the chaotic state gives one the possibility to drive a chaos back to a fully ordered state, thus opening new perspectives in the field of chaos control. In fact we wish to show all along this article that in order to understand the importance and the precise role of the defects one must make a refined and full description of their physical nature, i.e. of the topology of the "core". The defect then appears as a "localized instability" of an unstable state around a singularity. On these grounds one can understand more easily the role of defects either in structural phase transitions (symmetry-breakings) or at the onset of chaos.

2. Ordering, order-parameter, symmetries, organized fluids. We shall define ordered media and the description of tools used to characterize the ordering. Faults in this ordering are named defects which are singularities in the appropriate field. These definitions shall be used in the context of organized (ordered) fluids which can show ordering at two different scales and thus offer two different aspects of such singularities.

2.1. The order-parameter. An ordered medium can be described by a function $f(r)$ that varies continuously all over the space filled by this medium. The value it takes at every point is the order-parameter. The space of the possible values of this parameter is named the order-parameter space or the manifold of internal states. This order-parameter may be a scalar, a vector or a set of trihedral frames ([16], [17], [18]). If the orderparameter has everywhere the same value (i.e. the function $f(r)$ is constant) the medium is said to be uniform. In non-uniform media, on the contrary, the order-parameter varies 
continuously and in addition may show isolated ("localized") regions of lower dimensionality where it becomes null or even undefined. These singular regions are named defects. Not all distortions are singular and there, the topology gives tools to distinguish the real singularities. The full description of these non-uniform media must also take account of the symmetries. In fluids all symmetries are continuous, but in the case of hydrodynamic structures that we shall present in the sequel, obviously both continuous and discrete symmetries are involved. Defects originally, are defined as faults of stacking in the periodicity of discrete systems (any type of space or space-time periodic structure, e.g. solid crystals, lamellar structures, waves).

2.2. Groups-Symmetries. With an order parameter a group of transformations $G$ is associated that acts transitively on a space $\Sigma$ and also an isotropy subgroup $H$ for the reference order parameter, which expresses the symmetries of the medium. The order parameter space $\Sigma$ can be taken to be the space of cosets $G / H$ of $H$ in $G$. Thus in nematics $G$ is $S O(3)$ and the isotropy subgroup is the group $D_{\infty}$ of rotations about the molecular axis and $\Sigma=S O(3) / D_{\infty}=P^{2}\left(P^{2}\right.$ is the projective plane). A similar description applies to media with discrete symmetries. The group theoretical description is at the basis of the theory of phase transformations (in physics at thermodynamical equilibrium) and also by analogy in the bifurcations which are similarly, symmetry-breakings in out-of-equilibrium states. Thus in any transformation between homogeneous states the symmetry elements must be considered first. In what follows, we stress that they need also to be taken into account when the states are localized, i.e. around the singularities.

2.3. Organized fluids. Ordinary fluids are composed of molecules, the arrangement of which has neither orientational nor positional ordering in space. The symmetry of the state is therefore statistically maximal. On the contrary in organized fluids, there exists either a positional or an orientational ordering, or both. The most famous example of an organized fluid is the liquid crystal, in which in addition, the degree of ordering is temperature dependent. It is thus also named a thermotropic fluid and the different thermodynamic states of different symmetries, the mesophases. The isotropic state is met at a high temperature and the degree of ordering increases (the symmetry decreases) as the temperature is decreasing. The first ordered state is the nematic phase that we shall be concerned with in the following. There, the order-parameter which can be well related to a macroscopic quantity is represented by a binary vector, namely the director $\vec{n}$. Next, at lower temperatures in addition to the orientational ordering of the nematic there is a positional ordering in the mean direction of the molecules: the smectic phase.

Other types of fluids such as some biological ones [2] or diluted polymers or chemical compounds (lyotropic systems), show some spontaneous ordering related to the molecular conformation and with structures similar to the nematic or the smectic phases (e.g. soaps, microemulsions). Therefore many transitions between states of different symmetry, are met until the full solid crystal is obtained. The physical properties of organized fluids are tensorial because of the anisotropy introduced by the molecular conformation. The conformation of large assemblies of molecules is governed by elastic torques that maintain the ordering over large scales. The description of liquid crystal rely on a model adapted from the elasticity of continuous media, developed for usual crystals ([5], [21]). Those 


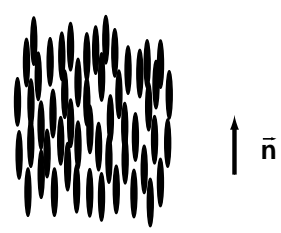

nematic state

orientational ordering but no positional ordering

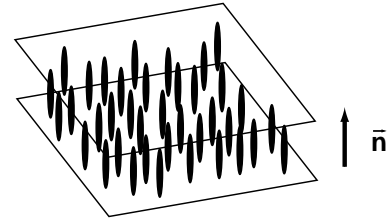

\section{smectic state}

both orientational ordering and positional ordering

Figure 1. The molecular ordering of the two main phases of a liquid crystal: the nematic (left) and the smectic (right). The director $\vec{n}(r)$ indicates the degree of orientational ordering (the order-parameter).

models are supplemented by a description of the special hydrodynamics which are coupled here, to the molecular orientation [7]. At thermodynamical equilibrium, use is rather made of the free-energy density. In the presence of any distortion due to flows or external actions (fields, boundary conditions on solid limits, ...) the total energy must be minimized expressing that an equilibrium has been attained. Such models allow one to express the average conformation on relatively large scales but are not adapted to describe precisely the topology of singularities down to the molecular scale. The fundamental model used to describe the free-energy variation $\delta F$ of a nematic resulting from a small distorsion of the director, is written as a function of the three basic elastic (Frank) constants $K_{i}[5]$ :

$$
\delta F=\frac{1}{2} K_{1}(\operatorname{div} \vec{n})^{2}+\frac{1}{2} K_{2}(\vec{n} \cdot \operatorname{curl} \vec{n})^{2}+\frac{1}{2} K_{3}[\vec{n} \times(\operatorname{curl} \vec{n})]^{2} .
$$

In all these compounds defects may occur on microscopic scales because of some external action, or improper (heterogeneous) boundary conditions, etc., that impose some torque on the molecular ordering. The fluid may react by adjusting continuously to the constraint or may develop, under circumstances that are not yet well-understood, singularities at some places. Those defects relax locally the constraint which can be either on large or on small scales.

Also, when subjected to the action of some external field such a fluid may also develop either static deformations or hydrodynamic structures which are ordered at macroscopic scales (of order the size of the sample itself). Then, defects of a new type may be created in these new structures at a macroscopic scale. In the following we shall describe defects occurring both at microscopic and macroscopic scales in the nematic phase of a liquid crystal. We shall show that the same topological tools of analysis can be used at both scales and that the two types of defects interact at the onset of the turbulent state.

3. Topological description of defects. The examples of the next two sections show that an ordered medium may possess defects, independently of its microscopic or macroscopic nature. A general topological classification of defects in continuous media has been constructed using the homotopy theory [26].

The main hypothesis is that the local physical state of the medium is described by an order parameter, which lies in some topological space $\Sigma$, the order-parameter space. We 
have seen that the local ordering of the (uniaxial) nematic liquid crystal is characterized by a director $\vec{n}$, element of the topological space $\Sigma=P^{2}$, the real projective plane. For two-dimensional nematics, $\Sigma=P^{1}=S^{1}$, i.e. the circle. The manifold $\Sigma$ is also called the manifold of internal states. Its dimension $m$ is usually different from the dimension $d$ of the domain $D$ occupied by the medium. The actual state of the medium associates with each point of $D$ a local state in $\Sigma$, defining a map $f: D \rightarrow \Sigma$.
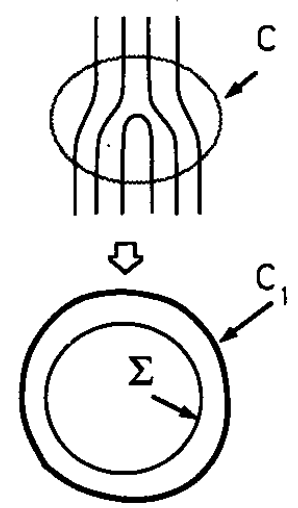

a )

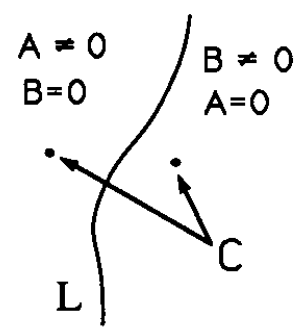

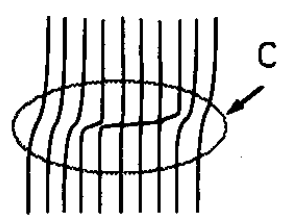

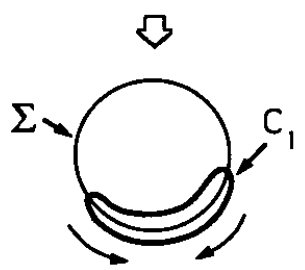

b )
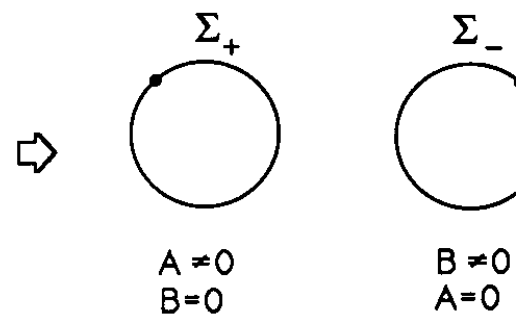
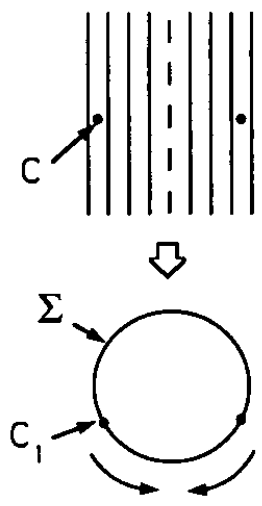

c)

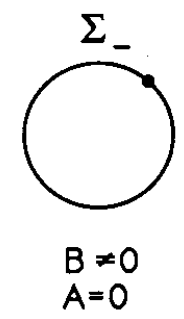

\section{d)}

Figure 2. Some examples of application of homotopy groups to experimental states:

a) A closed contour $C$ (i.e. the "sphere" $S$ ) around a point-defect in a 2-D space-periodic structure and its image $C_{1}$ in the manifold of internal states $\Sigma$. b) The image of $C$ encircling a phase modulation that is less than $2 \pi$ is homotopic to a point. Such an object is not a defect.

c) The image of a contour $C$ around a line-defect that corresponds in a periodic structure to a phase jump of $2 \pi$, is imaged by two points on the circle $\Sigma$. This defect is unstable because the two points can collapse. d) The line-defect is stable in the case of a parity symmetry-breaking that leads to a set of disconnected circles in the $\Sigma$ space.

This is the case of the two-variants structure of Oblique Rolls (zig-zag).

The map $f$ allows one to detect the presence of defects of any dimension $d^{\prime}$. For that we need to surround the defect by a sphere $S^{r}$ of dimension $r=d-d^{\prime}-1$. For instance, a linear defect $\left(d^{\prime}=1\right)$ in three-dimensional nematics $(d=3)$ is surrounded by a circle, the "sphere" of dimension $3-1-1=1$. Now the topological stability of the defect 
is expressed by saying that the restriction $\bar{f}=f_{\mid S^{r}}: S^{r} \rightarrow \Sigma$ cannot be continuously deformed into a constant map, which characterizes a uniform perfect medium. The defect is then associated with the (nontrivial) homotopy class of $\bar{f}$ and the possible topological types of defects are classified by the homotopy group $\pi_{r}(\Sigma)$. For instance, for the threedimensional (uniaxial) nematics, we have:

$$
\begin{aligned}
& \pi_{0}(\Sigma)=0 \\
& \pi_{1}(\Sigma)=Z_{2} \\
& \pi_{2}(\Sigma)=Z .
\end{aligned}
$$

The first relation shows that there is no stable wall in nematics. The second and the third relations show that linear and point defects are topologically allowed, but differently: there exists only one type of line-defect, while the point-defects are characterized by an index $s \in Z$. For nematics in the plane, we have:

$$
\begin{aligned}
& \pi_{0}(\Sigma)=0 \\
& \pi_{1}(\Sigma)=Z .
\end{aligned}
$$

The only topologically stable defects are the point-defects, which are characterized by an integer $s \in Z$, which represents the winding number of the director around the defect.

The homotopy theory is not merely a mathematical tool used to classify the defects in condensed matter. It gives also the possibility to make some predictions about the defect combinations. Since $\pi_{1}\left(P_{2}\right)=Z_{2}$, the linear defects in nematics, that is to say the disclination lines, are their own "opposite" defects and they can annihilate by pairs. Moreover it was shown that the non-commutativity of the fundamental group $\pi_{1}(\Sigma)$ represents an obstruction for the crossing of defect lines in dimension 3 (it is also assumed that $\pi_{2}(\Sigma)=0$ ) [22]. Biaxial nematics, for which the fundamental group is the (nonAbelian) quaternion group $Q$, provide an example of this case. However in many cases, the actual order parameter field must minimize some energy functional and the accessible states are far to be arbitrary, as it is assumed here. As a consequence, some topological defects are excluded or at least hardly observable. The topological analysis is also unable to describe the dynamical role of the defects, a role that we illustrate in the next section.

3.1. Topology and energetic considerations. Topological stability does not imply physical existence or stability. Reversely a topological instability does not mean that the defect is unrealizable. It can physically exist but transiently, or stabilized by some constraint, for instance boundaries conditions. The topological existence or stability indicates only the possibility for a given type of singularity to develop, but obviously it cannot give any information on the physical conditions necessary to create those singularities. The order parameter is associated with the director field $\vec{n}$, which can be coupled to external forces (electric, magnetic) or internal viscous torques. Those latter torques come from the velocity gradients which can be imposed from outside the medium. Another type of force are the elastic forces imposed for instance by the boundaries conditions (anchoring on solid surfaces). Therefore, singularities may be created by those torques provided that some barrier has been surmounted. This is the case for instance when strong velocity gradients are applied, and nematic threads are created. But the threads are unstable if they cannot 
be pinned onto the solid surfaces and they disappear by collapsing (reduction in size), although the associated singularities are topologically stable. Topological analysis simply provides a frame within which the evaluation of the energetic stability must be made to fully understand some of the physical properties of defects.

4. Singularities at microscopic scales. The nematic phase of liquid crystals was named so because of the presence of thin threads (from the Greek $\nu \epsilon \mu \grave{\alpha}$ ) immersed in the liquid, following a strong shaking of the fluid. These thin threads were soon recognized as defects in the field of the director $\vec{n}$ [6]. In fact, other localized objects having a character of defects or close to defects have been found and studied. However the situation is not totally clear at the present time concerning the relation between the observations, which are quite difficult, and the theory ([2], [3], [6], [15]).
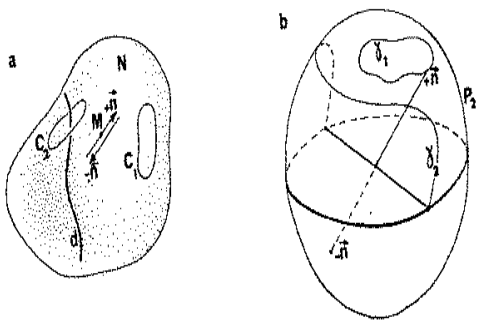

Figure 3. Topology of the core of a linear defect: the "disclination loop".

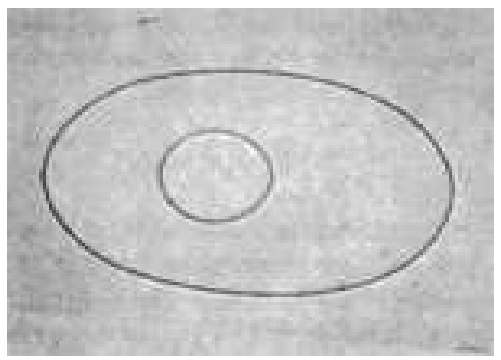

Figure 5. Two disclination loops are linear singularities in the nematic orientation.

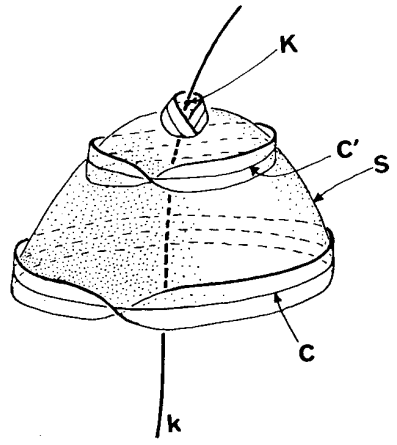

Figure 4. The formation of a disclination loop as a line of discontinuity by an "obstruction principle" ([2], [3]).

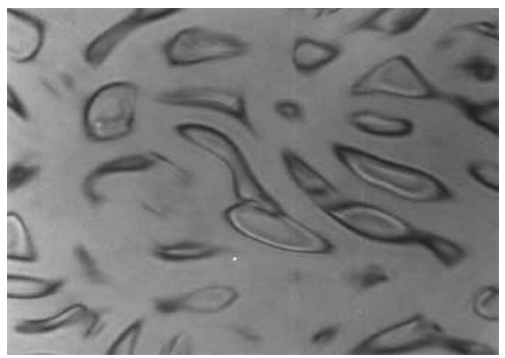

Figure 6. Linear singularities: disclination loops close to onset of turbulence.

4.1. The linear defects (the "nema"). They are developed easily in the bulk in the presence of velocity gradients. When present, the threads form loops (Fig. 5) which shrink in time and usually vanish out. However those line-defects may persist when they are pinned by some way to the limiting solid boundaries. At these surfaces point-defects appear. From topological analysis it is shown that since $\pi_{1}\left(P_{2}\right)=Z_{2}$ there is only one class of non-removable linear singularities. These are named disclination lines because 
the director $\vec{n}$ rotates by $\pi$ as the singular line is encircled. Bouligand has described the topology of the director by using a Möbius stripe encircling the line ([2], [3]) and which would shrink as the line is getting closer (Fig. 3, 4). We shall be concerned in this article, by loops created by the velocity gradients at the onset of hydrodynamic turbulence (Fig. 6).

4.2. The point-defects. Point-defects are never seen as isolated singularities inside the bulk. They can only appear attached to lines that interact, or when the boundaries exert well-defined torques. The torques must have a given symmetry and a sufficient value, and usually in ordinary nematics such conditions are possible when the solid boundaries are at small distances from each other (Fig. 10). Singular points also may appear isolated on the solid boundaries and under circumstances that are not yet well elucidated (Fig. 9). The point-defects commonly observed are those terminating a disclination loop on a surface where they become pinned (Fig. 8). In that case, we may remark that the topological description is not adapted to such a situation where boundaries have to be involved. Next, at the surface one recovers a classical situation of singularities of a director field in a 2-D space. There, the singularities are characterized by their index (Fig. 7).

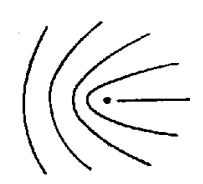

$s=\frac{1}{2}$

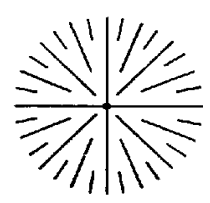

$s=1, c=0$

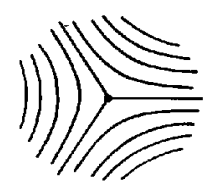

$s=-\frac{1}{2}$

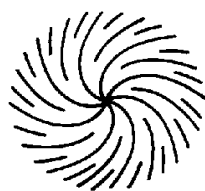

$s=1, c=\pi / 4$

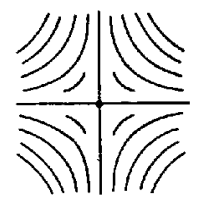

$s=-1$

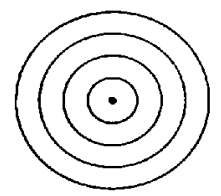

$s=1, c=\pi / 2$

Figure 7. Molecular orientation (director field) in the neighborhood of some types of point-singularities in a 2-D space (see [5]).

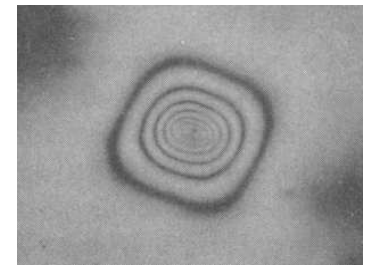

Figure 8. A punctual singularity. Usually such a point-defect is present only at the solid boundaries limiting the nematic.

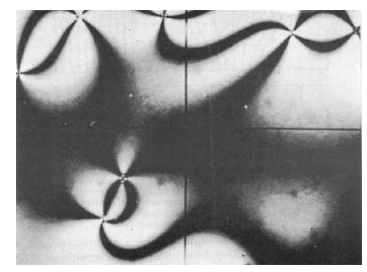

Figure 9. Optical pattern due to the discontinuities produced by punctual singularities in the ordering of a nematic.

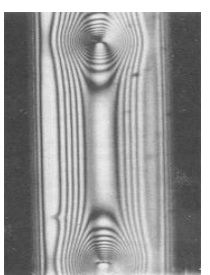

Figure 10. Singularities of opposite sign in a nematic liquid crystal confined inside a capillary. 
In the following, those point-defects will not be considered since they do not appear in the bulk and even if they were created transiently, they would immediately interact with the lines and disappear.

\section{Singularities at macroscopic scales}

5.1. Ordered macroscopic structures. Organized fluids as well as ordinary fluids may develop hydrodynamic flow structures when they are subjected to an appropriate external constraint. For instance by applying a vertical thermal gradient or an electric field across a layer of fluid, one creates well-ordered convective structures [10], periodic in time and showing also in some cases, a periodicity in time (travelling waves). Organized fluids are more sensitive to such constraints because of their intrinsic anisotropy. In addition the anisotropy imposes extra symmetry elements that help stabilizing some states contrarily to the case of usual fluids. In a liquid crystal subjected to a transverse electric field it has been shown that a series of symmetry-breakings occur from the rest state, up to the fully turbulent state [10] (Fig. 11). Those states are of decreasing symmetry and the transitions are named bifurcations. The characteristics of the bifurcations are universal ones and the number of such states is finite and corresponds to all possible symmetries in a plane, since until and before the turbulence, the mid-plane of the layer keeps being a symmetry plane for the flow, thus eliminating the third dimension. At rest, the molecules are aligned by an experimental procedure, along say the $\vec{x}$ direction. The first convective structure corresponds to cylindrical rolls whose axis is aligned perpendicular to $\vec{x}$, i.e. along $\vec{y}$. They are named Normal Rolls and may be represented by their vortex lines aligned along $\vec{y}$.

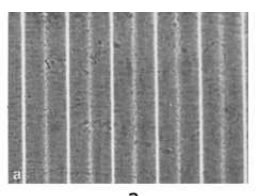

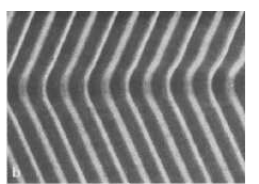

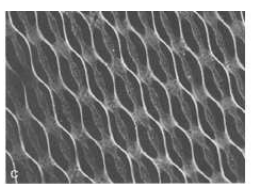

c

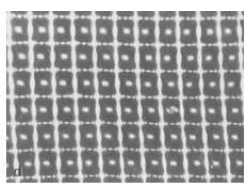

d

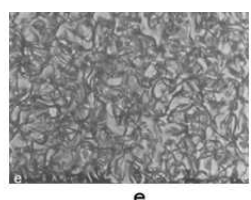

e

Figure 11. The cascade of symmetry-breakings in the convection structures developed in a liquid crystal, from the rest state to turbulence: a) the Normal Rolls, b) the two-variant Oblique Rolls, c) the bi-modal varicose, d) the rectangular bimodal, e) the turbulent state.

5.2. Defects of ordered macroscopic structures. In all these structures, defects may occur on macroscopic scales. As regards the topology, those macroscopic hydrodynamic structures represent the case of ordered media with broken translational symmetry. The continuous translational invariance is replaced by a discrete (periodic) translational invariance. The topology analysis developed for continuous invariance has been generalized to these discrete media [14]. As already mentioned, the whole system is symmetrical with respect to the median plane of the layer and therefore can be considered as a 2-D system. Two types of point-defects are possible in structures that break the continuous translational invariance: the rotational and the translational dislocations. But the symmetry of the medium (anisotropy) which breaks the rotational invariance allows only the second type to exist. Such a defect can be characterized by its winding number $N$ which measures the phase jump along a closed contour encircling the singularity. In such systems 
only numbers $N=1$ are observed (Fig. 12a). In the plane, the order parameter is represented as a complex number $A(r)=A_{0}(r) \exp i \Phi(r)$. The singularity is defined as the point $P$ where the amplitude of the order-parameter vanishes $A_{0}(r)=0$ and the phase $\Phi(r)$ is undetermined. The close vicinity of $P$ is named the core (see e.g. Fig. 12a,b) and corresponds here to a matching solution connecting the singularity with the unperturbed medium. As we shall see in the next section, the topology of the core and its surroundings reflects the symmetry elements of the unstable (of lesser symmetry) states. This feature gives the defects properties that allows one to understand their role in the structural transformations occurring when the external constraint is varied, and especially below and inside the chaotic state. The linear defect is topologically unstable in the Normal Rolls, but exists in the Oblique Rolls state which has a parity symmetry and is composed of two symmetrical variants (Fig. 12c,d). In fact, it is found that the topology of these line is somehow similar to that of the point-defects. The line-defects represent a linear stacking of point-defects as it can be seen on Fig. 12d.

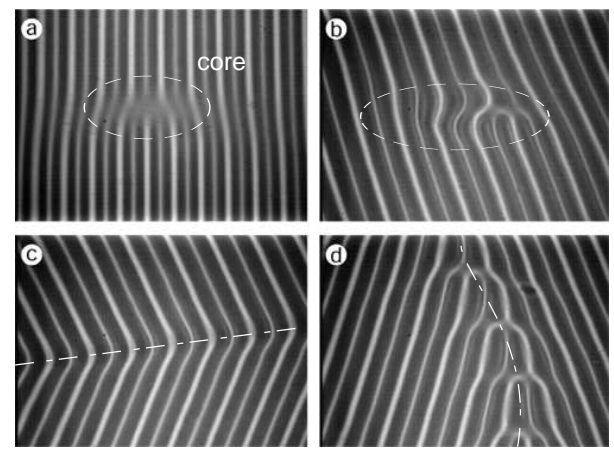

Figure 12. Point-defects in hydrodynamic periodic structures:

a) in the Normal Rolls (edge-dislocation); b) in the Oblique Rolls the core

is asymmetrical. The core is approximately delineated (dashed curve).

Line-defects c)-d) two equivalent linear defects (dash) separate the two variants of the Oblique Rolls. Although they appear differently, they have the same topology.

6. The role of defects in the structural transformations. We shall examine in the following the role of the singularities successively inside homogeneous, heterogeneous, chaotic and turbulent space-time states.

6.1. Inside homogeneous states. Although the defects had been observed for long times and in many circumstances, the mechanisms for their creation and their interaction with the underlying states where not studied and even not considered. They were just taken as incidental "imperfections". The importance of defects became apparent only after precise studies of their topology in relation with the physics of the basic state were made ([13], [12]). There, it appears that for instance, in the convective structures of a liquid crystal, the defects are created following the application of sudden steps of the constraint by a mechanism of localization of an instability through a process analogous to a focussing. 


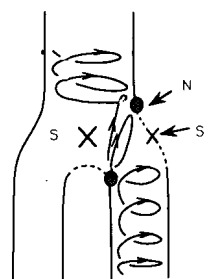

dislocation

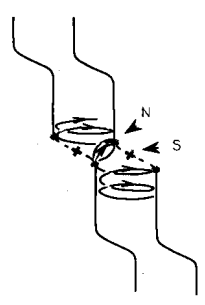

varicose

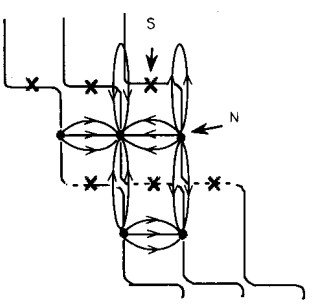

bimodal

Figure 13. The hydrodynamic singularities (stagnation points) associated with a local symmetry-breaking, first inside the core of a defect, then in the varicose state, next in the bimodal, as deduced from the experimental tracing of the flow streamlines. The singularities of the velocity field are associated with the local symmetry-breaking (creating here the pinching by the second mode of the bimodal).

Next, the instability represents precisely the most unstable state for the average conditions. It is characterized by a lower symmetry. Therefore, a defect is a singularity in the order parameter which is associated with a localized symmetry-breaking. The topology of a defect (be it a point or a line) is experimentally deduced from the tracing of streamlines within the flow structure. In our case a point-defect (also named a dislocation) contains around its core the symmetry elements of the rectangular bimodal state (see Fig. 11), which itself is characterized by singularities in the flow field structure, under the form of stagnation and nodal points. Therefore, one can give a simple description of the flow field inside those states based on the stability of a vector field which is sufficient to represent the whole physics. There, the singularities are created by "destabilizing" successively the singular objects, as the external constraint is increased. Firstly, the plane with zero velocity (the rest state) destabilizes into singular lines which represent for instance the vortex lines of the first structure of convective rolls (Fig. 14).

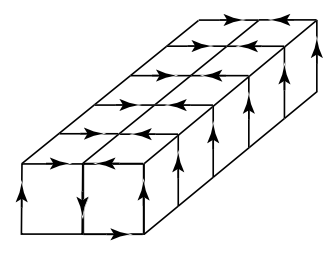

a

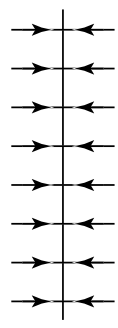

b

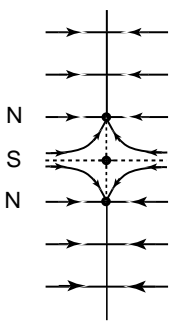

C

Figure 14. The instabilities in a vector field (either the velocity or vorticity field) produce first, (a) singular lines, next singular points. At each step the dimension of the singularity is reduced.

Secondly, the singular lines destabilize under a further increase of the constraint giving singular points, the stagnation and node points (Fig. 15). The whole sequence of periodic structures towards the full chaotic state is then described as a sequence of structural transformations of periodic ensembles of singularities (Fig. 16). It has been shown experimentally that a point-defect is created by a localized instability of varicose-bimodal. 


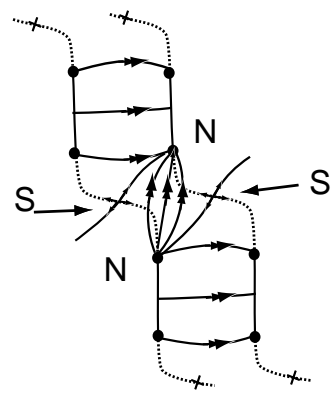

a

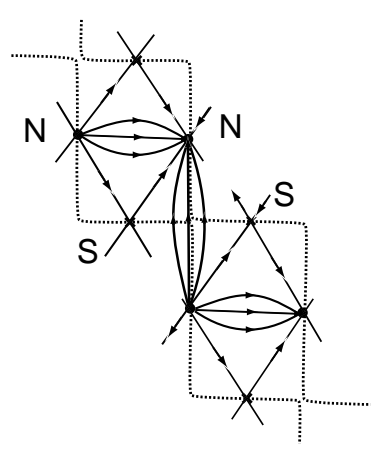

b

Figure 15. The stagnation points resulting from the instability of the singular lines in the case of the varicose (a) and the bimodal (b) velocity fields. The experimental streamlines do conform to this representation and $\mathrm{N}$ are the nodal points (sinks) and $\mathrm{S}$ are the stagnation points (saddle), see Fig. 14.

Therefore the singular elements found in this unstable state are present in the core of the defect as it is demonstrated by the experiment. From these results it follows that the role of defects in the bifurcations to lower-symmetry states can be understood on these simple grounds. Those bifurcations can be mediated by the defects which act as germs for the nucleation of the lower-symmetry states. On the other hand, the defects have a crucial importance in the spatio-temporal chaotic states where they are one of the essential attractors. The other type of singularity which is allowed in those states is the line-defects. They also are essential in the dynamics of the bifurcations as well as those of the chaotic state. It can be shown both experimentally and by topological arguments that these lines may reduce to point-defects. Therefore their role is essential as far as the long scales are concerned, i.e. whenever domains with different order-parameter value are possible.

6.2. Inside heterogeneous (chaotic) states. There, both types of defects have a role which depends not only on their scale but also on their "structure". In effect, up to now the order-parameter that was associated with the state was not only constant in space (homogeneous states), but also constant in time (stationary). It has been recently found that the chaotic state consists of a few attractors that are explored randomly by the system [1]. Those attractors are not necessarily "chaotic" by themselves, and indeed here, are fixed points, each one associated with a space-periodic state. In addition there exists a periodic time-dependent state (limit cycle) associated with the point-defects. The chaotic character comes from the fact that the defects are randomly distributed in space and out of phase in time (de-synchronized).

6.3. Inside the fully turbulent state. The full turbulence is characterized by the absence of any ordering (correlation) at any scale down to the microscopic scales. In an organized fluid the ultimate scale is the molecular length and at that scale, the absence of ordering is indicated by the presence of the singularities (the defects) described in Section 3. And indeed, the only structures that appear in the turbulent state are the disclination loops whose density is such that the fluid becomes turbid (Fig. 11e). These 


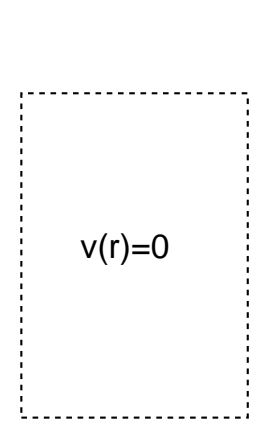

a

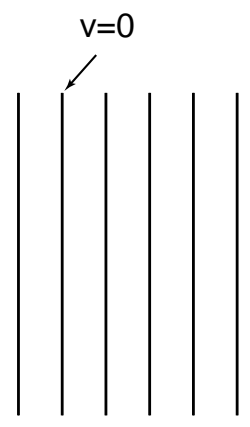

b

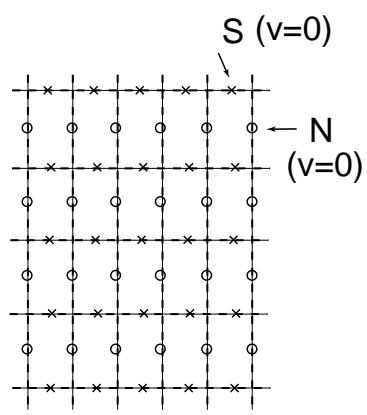

C

Figure 16. The sequence of instabilities in a vector field (here either the vorticity or a velocity component). At rest in the whole plane $v=0$ everywhere (a). It is unstable against a periodic distribution of lines (b) where again $v=0$. Next, the lines become unstable against a periodic distribution of points with alternate "charge"

in both directions (c). At each step the dimension of the singularity is decreasing. This sequence is to be compared with that of Fig. 11.

loops are created as soon as the velocity gradients become high enough to impose a localized breaking in the molecular ordering. The experiment shows optical de-correlations down to scales of order the light wavelength, i.e. the ultimate length attainable experimentally. In the case of convection, the ordered macroscopic structures persist until they become disorganized at scales of order the convection scale (here some $10 \mu \mathrm{m}$ ). In a first step, only the macroscopic defects are created, and their density increases in order to decrease the space scales, as the constraint increases. This corresponds to a very high external constraint. Therefore the velocity gradients become now high enough to create defects at microscopic scale, but their extent, and therefore their density, is limited by the convection scales. At some higher value of the constraint however, the gradients around the loops are greater than those associated with the convective flow and the density of the loops starts increasing while the macroscopic ordering disappears. The fully turbulent state is attained and there is no more ordering at either the macroscopic or the microscopic scales.

\section{Controlling a spatio-temporal chaos through the synchronization of de-}

fects. Once a spatio-temporal chaos has been analyzed it is possible to control it in some manner by acting on one or more of its attractors.

7.1. Temporal forcing. A chaos is characterized by disorder both in time and in space (Fig. 17). It was recognized in the system under study that the time-dependent state is associated with the point-defects [1]. It comes in fact from the existence of a new mode which cannot be evidenced optically but which interacts with the basic state of Oblique Rolls. And precisely this state is present inside the core of a defect and interacts with the basic purely spatial mode. The interaction results in a oscillation in time of the position of the core [1]. Those defects are de-synchronized because they are randomly spread. An external forcing is applied by modulating the external constraint periodically in time with 


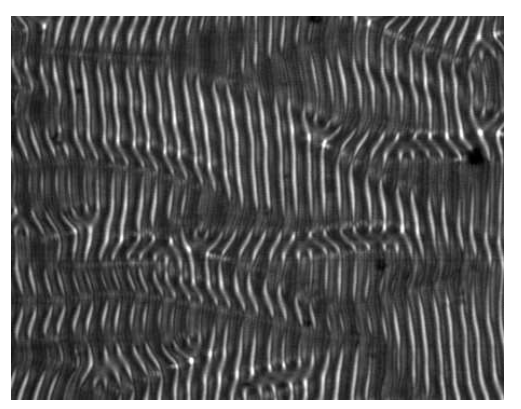

Figure 17. A chaotic state ("weak chaos") is characterized by the presence of a small number of attractors, seen here as portions of structures still ordered on small scales and a time-dependence associated with the motion of both point and line-defects. There is an incoherent slippage of the phase from place to place. The structures seems to vacillate randomly in space and in time.

a period twice that of the oscillation of the defects. New defects are created which are now synchronized with the constraint and annihilate the previous ones. When the synchronized defects are the only ones left they mutually interact now in space and aggregate. Since the defects have the symmetry elements of the otherwise unstable state, they shall form precisely this ordered state as the forcing increases. However one has to take into account the new symmetry introduced by the forcing (breaking of continuous time invariance), and the new structure includes those new symmetry elements [1] (Fig. 18). This is an example of defect-mediated structural transformation.
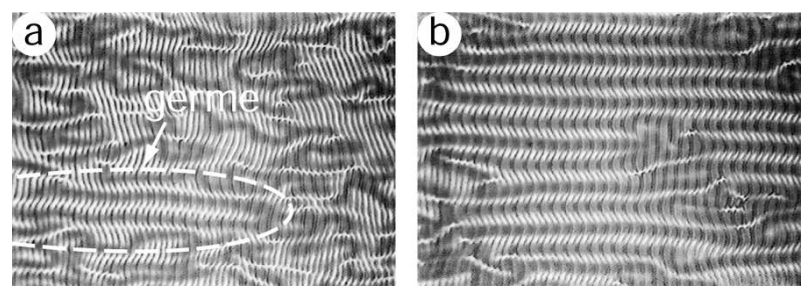

Figure 18. A time-dependent (periodic) modulation of the control parameter is applied to a chaotic state (a). The control of the chaotic attractor starts by the nucleation of germ in which the defects are synchronized. After some time the whole space becomes fully ordered both in time and in space (b).

7.2. Spatial forcing. The spatial forcing consists of applying through a specific device, a spatial modulation of the otherwise homogeneous constraint. In a very simple case this modulation is periodic in one direction and constant in the orthogonal one, with characteristics close to the simplest realizable unstable state. For instance, the geometrical quantities (period, orientation) are chosen to be close to those of one of the attractors defining the chaotic state [8]. In fact, such an experiment is similar to the forcing of oscillators used long ago to study the response of oscillators and deduce their properties.

An important parameter is the modulation-depth $m$ which is the ratio of the forcing amplitude versus the external constraint amplitude. The second parameter is the geometrical period $k(r)$. For values of $m$ beyond some well-defined threshold the chaos 

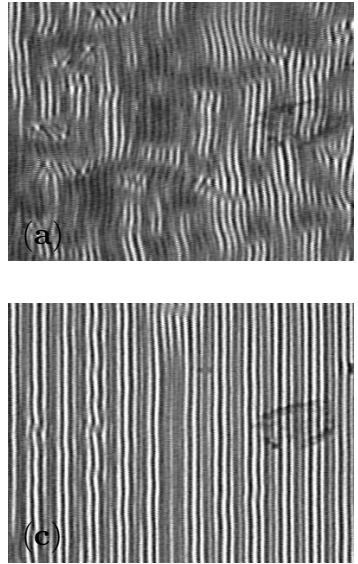
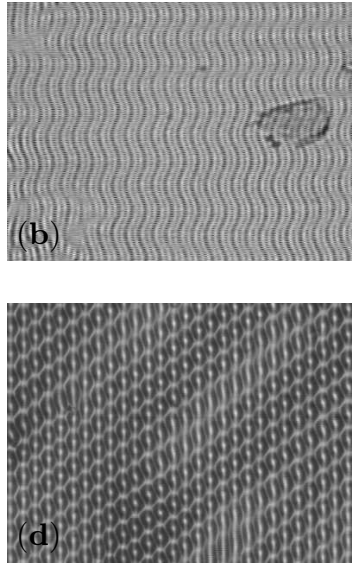

Figure 19. a) The initial space-time chaotic state, b) by imposing a modulation in time of the external constraint the defects are eliminated and the final state is periodic both in time and in space. c) a stationary homogeneous state obtained by imposing a spatially-modulated forcing with appropriate wave-vector.

is suppressed (chaos reduction) and replaced by a perfect periodic stationary structure (Fig. 19). The forcing by imposition of a regular order-parameter state, prevents all defects to develop by incidental localization in space. Next, it forces the attractor to keep around the imposed fixed-point ([8], [9]).

8. Conclusion. Defects arising in ordered media play a prominent role as regards the stability of the embedding state. They can mediate any state transformation occurring under an external constraint in the case of out-of-equilibrium states, or as the temperature is varied in the case of thermodynamic equilibrium. Their properties can be well understood by analyzing the fine structure of the neighborhood of the singularity. They appear thus as localized instabilities and their neighborhood has the features of the unstable state. Their topological properties are thus easily related to their physical role. In conclusion, defects represent local symmetry-breakings, the study of which can be made in the simplest cases using their homotopy group. Their role is particularly evidenced in the chaotic state or even in the full turbulence.

\section{References}

[1] A. Belaidi, Ph.D. thesis, Univ. Paris XI, Orsay, France, 1997.

[2] Y. Bouligand, Geometry and topology of defects in liquid crystals, in: Physics of Defects (Les Houches, 1980), R. Balian et al. (eds.), North-Holland, Berlin, 1981, 665-711.

[3] Y. Bouligand, Recherches sur les textures des états mésomorphes . .., J. Physique 35 (1975), 215-236.

[4] F. Falk, Landau theory of martensitic phase transitions, J. Physique 43-C4 (1958), 3-15.

[5] F. C. Frank, On the theory of liquid crystals, Disc. Faraday Soc. 25 (1958), 19-28.

[6] G. Friedel, Les états mésomorphes de la matière, Annales de Physique 18 (1922), 273. 
[7] P. G. de Gennes, J. Prost, The Physics of Liquid Crystals, Clarendon Press, Oxford, 1993.

[8] M. Henriot, Ph.D. Thesis, Univ. Paris XI, Orsay, France, 2001.

[9] M. Henriot, R. Ribotta, Forçage spatial d'états non linéaires, in: Rencontres du non linéaire, Y. Pomeau and R. Ribotta (eds.), Paris Onze ed., Orsay, 2000, 49-54.

[10] A. Joets, R. Ribotta, Hydrodynamic transitions to chaos in the convection of an anisotropic fluid, J. Physique 47 (1986), 595-606.

[11] A. Joets, R. Ribotta, Localized bifurcations and defect instabilities in the convection of a nematic liquid crystal, J. Statist. Phys. 64 (1991), 981-1005.

[12] A. Joets, R. Ribotta, X. D. Yang, Defects of stationary convective structures in a nematic, in: Nematics (Orsay, 1990), J. M. Coron et al. (eds.), NATO Adv. Sci. Inst. Ser. C Math. Phys. Sci. 332, Kluwer, Dordrecht, 1991, 189-194.

[13] A. Joets, X. D. Yang, R. Ribotta, Singularities in the transitions to chaos of a convective anisotropic fluid, Physica 23D (1986), 235-239.

[14] M. Kléman, Relationship between Burgers circuit, Volterra process and homotopy groups, J. Physique Lett. 38 (1977), L-199-L-202.

[15] M. Kléman, Points, Lignes, Parois, Editions de Physique, Orsay, 1977.

[16] M. Kléman, L. Michel, Spontaneous breaking of Euclidean invariance and classification of topological stable defects and configurations of crystals and liquid crystals, Phys. Rev. Lett. 40 (1978), 1387-1390.

[17] N. D. Mermin, The topological theory of defects in ordered media, Rev. Modern Phys. 51 (1979), 591-648.

[18] L. Michel, Classification topoogique des défauts et des configurations des milieux ordonnés, in: Physics of Defects (Les Houches, 1980), R. Balian et al. (eds.), North-Holland, Berlin, 1981, 364-384.

[19] L. Michel, Symmetry, defects and broken symmetry. Configurations. Hidden symmetry, Rev. Modern Phys. 52 (1980), 617-651.

[20] F. R. N. Nabarro, Theory of Crystal Dislocations, Clarendon Press, Oxford, 1967.

[21] C. W. Oseen, The theory of liquid crystals, Trans. Faraday Soc. II 29 (1933), 883-899.

[22] G. Poénaru, G. Toulouse, The crossing of defects in ordered media and the topology of 3-manifolds, J. Physique 38 (1977), 887-895.

[23] R. Ribotta, Defects-mediated bifurcations in the convection of a nematic liquid crystal, in: Nonlinear and Chaotic Phenomena in Plasmas, Solids and Fluids (Edmonton, 1990), W. Rozmus and J. A. Tuszyński (eds.), World Scientific, River Edge, 1991, 596.

[24] R. Ribotta, A. Joets, Defects and interactions with the structures in EHD convection in nematic liquid crystals, in: Cellular Structures in Instabilities, J. E. Wesfreid and S. Zaleski (eds.), Lecture Notes in Phys. 210, Springer, Berlin, 1984, 249-262.

[25] D. Rogula, Large deformations of crystals, homotopy and defects, in: Trends in Applications of Pure Mathematics to Mechanics (Lecce, 1975), G. Fichera (ed.), Pitman, London, 1976, $311-331$.

[26] G. Toulouse, M. Kléman, Principles of a classification of defects in ordered media, J. Physique Lett. 37 (1976), L-149-L-151.

[27] G. E. Volovik, V. P. Mineev, Investigation of singularities in supefluid $\mathrm{He}^{3}$ in liquid crystals by homotopic topology methods, Zh. Èksp. Teoret. Fiz. 72 (1977), 2256-2274 (Russian); English transl.: Soviet Physics JETP 45 (1977), 1186-1196.

[28] X. D. Yang, A. Joets, R. Ribotta, Localized instabilities and nucleation of dislocations in convective rolls, in: Propagation in Systems far from Equilibrium, Synergetics, J. E. Wesfreid et al. (eds.), Springer, Berlin, 1988, 194-203. 